\title{
INVESTIGACIONES
}

\section{Las emociones como dificultad didáctica en la enseñanza de prácticas motrices: Los problemas afectivos derivados del contacto. Conceptualización}

\author{
Emotions such as didactical difficulty teaching physical education. \\ Emotional problems related to contact: Conceptualization \\ José Ignácio Salgado López $z^{a}$ José Andrés Sánchez Molina ${ }^{b}$, \\ $M^{a}$ Inmaculada Canales-Lacruz \\ ${ }^{a}$ Profesor de Ensino Secundario de la Xunta de Galiza. IES Leliadoura (Ribeira -A Coruña). \\ inhaqui@edu.xunta.es \\ ${ }^{b}$ Profesor Titular de la Universidade da Coruña. \\ jasanmol@udc.es \\ ${ }^{c}$ Profesora Titular de Universidad de la Universidad de Zaragoza. \\ bromato@unizar.es

\section{RESUMEN} \\ Las dificultades didácticas asociadas al contacto en las prácticas motrices son una realidad muchas veces \\ olvidada total o parcialmente en los procesos de enseñanza-aprendizaje de ese tipo de prácticas. Esta situación \\ viene determinada por el carácter multidimensional del concepto y, en parte, por su origen empírico, lo que \\ implica una gran diversidad terminológica. El presente trabajo pretende aportar una propuesta justificada para la \\ conceptualización de los problemas afectivos derivados del contacto.
}

Palabras clave: miedo al contacto, miedo al balón, miedo a la caída, vergüenza, asco.

\section{ABSTRACT}

The didactic difficulties associated to contact in motor tasks are often partially or fully forgotten in teachinglearning processes of this type of practice. This situation is determined by the multidimensional nature of the concept and, in part, by its empirical origin, which has led to a great terminological diversity. This paper provides a justified proposal for the conceptualization of emotional problems related to contact.

Key words: fear of physical contact, fear of falling, fear of the ball, embarrassment, disgust. 


\section{INTRODUCCIÓN}

En el proceso de enseñanza-aprendizaje de prácticas motrices existe una continua interacción entre las personas participantes. El simple contacto entre partícipes del acto didáctico puede resultar molesto para algunos, pero en la enseñanza de las prácticas antes citadas es evidente que el contacto contempla situaciones más allá de dicha interacción, ampliándose a los producidos con el suelo y/o los objetos (por ejemplo un móvil). Hay estudios que muestran la relación entre la aparición de conductas desajustadas y los contactos producidos en las tareas motrices empleadas en procesos de enseñanza-aprendizaje (Canales y Casedas, 2018; Salgado López, 2014). Sin embargo, apenas se encuentra en la bibliografía referencias a estos problemas de interés para la praxis en la enseñanza de prácticas motrices.

Pensamos, por tanto, que será necesario clarificar primero nuestro objeto de estudio, que, como acostumbra a suceder en nuestro campo epistemológico, nace empíricamente de las experiencias de los profesionales. Esto implica una multiplicidad de pareceres que no ayudan a la resolución del problema.

\section{OBJETO DE ESTUDIO}

Las emociones que emanan de las situaciones de contacto surgidas en situaciones de aprendizaje motor pueden considerarse una dificultad didáctica, siempre que den como resultado una conducta motriz desajustada, o si se quiere, un problema a superar para lograr dicho aprendizaje. Esto supone adentrarse en la esfera subjetiva del individuo para poder comprender, aun parcialmente, la realidad de las conductas motrices dentro de los procesos de enseñanza-aprendizaje de prácticas motrices. Es importante señalar que este objeto de estudio obliga a centrarnos en la parte subjetiva del concepto "conducta motriz" (Parlebas, 2001), superando la visión mecanicista.

Una de las características del objeto de estudio es su indefinición a nivel científico y su amplia constatación a nivel empírico. Así, es común hablar de miedo al balón por ejemplo al aprender a recibir un pase de baloncesto o un golpeo en voleibol. Asimismo, es común hablar del miedo a la caída también en voleibol o en gimnasia deportiva; igualmente es común la expresión miedo al contacto para referirse al llamado contacto físico entre personas. En este último caso se puede relacionar, dependiendo del campo de estudio, con aspectos de mantenimiento de la integridad física o con los espacios personales que había definido Hall (2003).

\section{DIMENSIONES DEL OBJETO DE ESTUDIO}

La indefinición referida parece ser fruto de la naturaleza pluridimensional del problema donde podemos encontrar:

- aspectos relacionados con el mantenimiento de la integridad física, que en el ámbito de prácticas motrices podrían asociarse al miedo a lesionarse;

- aspectos relacionados con la comunicación y/o ciertos aspectos psico-morales o socio-culturales que pudieran existir según el ambiente en el que el sujeto se desarrolle; 
- una dimensión intermedia, de orden filogenético, resultado del solapamiento de los aspectos anteriores, relacionada con el concepto de "territorialidad" descrita por Hall (1989, p. 58) y que enmarcarían las llamadas "esferas o espacios personales" (Hall, 2003). Estas esferas tratan de explicar el uso social del espacio siendo aceptado y/o adaptado por muchos autores (Bernard, 1985; Canales, 2006; Davis, 1987; Descamps, 1990; Fast, 1994; Hernández Moreno, 1994; Montagu y Matson, 1989; Pease y Pease, 2006; Sommer, 1974).

\section{CONTACTO Y EMOCIONES}

Anteriormente se han descrito las relaciones existentes entre el contacto y los aspectos culturales (Mújica-Johnson y Salgado López, 2021). Junto con estos, podemos señalar que, en las prácticas motrices aparece otros aspectos que convierte al contacto en fuente de ansiedad y desasosiego. Estos otros se refieren a las emociones que surgen a raíz de experiencias negativas de contacto, en las que la imagen, la integridad física, etc., puede verse comprometida. Muestra de ello son las abundantes referencias a este hecho en la bibliografía consultada y que referenciaremos a lo largo de este trabajo.

En esta última década se ha despertado un elevado interés investigador por el estudio de las emociones dentro del ámbito de la enseñanza de las prácticas motrices como se recoge de las revisiones de Mujica-Johnson, Orellana-Arduiz y Concha-López (2017) y Martínez-Martínez y Valero-Valenzuela (2019). Ahora bien, ya la primera de ellas resalta que los trabajos publicados se centran, fundamentalmente, en la descripción de la vivencia emocional suscitada por las tareas motrices y cómo esta afecta al bienestar de los implicados en las clases de EF. Sin embargo, en este trabajo intentaremos describir las intrincadas relaciones entre las distintas variantes de contactos producidos en las situaciones de aprendizaje de prácticas motrices, las emociones que cada una de ellas puede generar y como éstas pueden influir en el propio aprendizaje motor del sujeto. Para concretar más el campo de estudio nos centramos únicamente en aquellas suscitadas en relación con el contacto, puesto que, en gran medida, serán modulables por la acción del docente al diseñar las tareas motrices.

Damasio (2006, p. 179) define la emoción como "la combinación de un proceso evaluador mental, simple o complejo, con respuestas disposicionales a dicho proceso, la mayoría dirigidas hacia el cuerpo propiamente dicho, que producen un estado corporal emocional, pero también hacia el cerebro (...) que producen cambios mentales adicionales". Así, este autor diferencia emoción de sentimiento, que sería la "experimentación" y la "percepción de todos los cambios que constituyen la respuesta emocional". Además, recoge como "emociones universales básicas" las centradas en la "Felicidad, Tristeza, Ira, Miedo y Asco" y como secundarias, pequeñas "variaciones sutiles" de las cinco anteriores, entre ellas, por ejemplo, cita el "pánico" y la "timidez" como variaciones del miedo.

De forma similar, Bisquerra Alzina (2003, p. 12) define el concepto de emoción como "un estado complejo del organismo caracterizado por una excitación o perturbación que predispone a una respuesta organizada".

Si bien el proceso emocional es mucho más complejo, como pone de manifiesto Damasio (2006), para Bisquerra Alzina (2003), de forma resumida, la emoción se produce de la siguiente forma: 
Estudios Pedagógicos XLVII N 3: 167-190, 2021

LAS EMOCIONES COMO DIFICULTAD DIDÁCTICA EN LA ENSEÑANZA DE PRÁCTICAS MOTRICES: LOS PROBLEMAS AFECTIVOS DERIVADOS DEL CONTACTO. CONCEPTUALIZACIÓN

- Unas informaciones sensoriales llegan a los centros emocionales del cerebro.

- Como consecuencia se produce una respuesta neurofisiológica.

- El neocortex interpreta la información.

En todo caso, ambos autores asumen la influencia que la emoción tiene en la consciencia y actitud corporal, relacionas con lo que Damasio (2006) denominada como "estado corporal emocional", y LeDoux (2012) "emocional estate". Por su parte, Bisquerra Alzina (2003) representa el concepto de emoción a partir de los componentes "neurofisiológico", "comportamental" y "congnitivo" que influyen directamente en una "predisposición para la acción" determinada.

\section{CLASIFICACIÓN DE LAS EMOCIONES}

Profundizando en el estudio de las emociones, Lazarus (2000, p. 232) clasifica las emociones en "«negatively toned emotions,» such as anger, anxiety, fright, sadness, guilt, shame, envy, jealousy, and disgust" y "«positively toned emotions» such as relief, hope, happiness/joy, pride, love, gratitude, and compassion". Ahora bien, matiza que, aunque la clasificación parece otorgar a cada emoción un valor positivo o negativo, bajo ciertas condiciones una emoción del grupo negativo puede tener un efecto positivo, citando como ejemplo "when the negative judgment motivates an athlete to seek a more constructive pattern of attention and concentration or to abandon a losing strategy for one that works". En esta línea, por ejemplo, Damasio (2006) considera el miedo como necesario para la supervivencia del ser humano, en tanto que representa un sistema de alerta ante peligros para la integridad del individuo. En el caso de la vergüenza, La Taille (2002, p. 14) mantiene que "a vergonha deve ser um sentimento fundamental, não apenas para a conduta moral dos homens, mas também para o desenvolvimento moral da criança e do adolescente". En consecuencia, siguiendo a los autores anteriores, podemos decir que las emociones son imprescindibles.

Adentrándonos en la concreción de un repertorio de emociones Lazarus (2000) expone que cualquier propuesta es argumentable, en lo que coincide con otros autores (Bisquerra Alzina, 2000; Damasio, 2006), citando como ejemplos de cada uno de los grupos las siguientes: anger, anxiety, fright, guilt, shame, sadness, envy, jealousy, happiness, pride, relief, hope, love, gratitude, compassion.

En parte derivada de la anterior y sin querer entrar en la complejidad de la descripción de cada una de ellas y sus variantes, Bisquerra Alzina (2000) recoge los siguientes tipos de emociones, categorizándolas en:

- Emociones positivas: emociones placenteras, agradables, se experimentan al lograr una meta. Representadas por la alegría, el humor, el amor y la felicidad y sus distintas variantes.

- Emociones negativas: emociones desagradables, que se experimentan cuando se bloquea una meta, ante una amenaza o una pérdida. Se pueden concretar en la ira, el miedo, la ansiedad, la tristeza, la vergüenza y la aversión, además de las distintas manifestaciones de cada una de las anteriores.

- Emociones ambiguas: aquellas que pueden considerarse emociones positivas o negativas en función de las circunstancias: sorpresa, esperanza, compasión. 
- Emociones estéticas: se dan cuando se reacciona emocionalmente ante ciertas manifestaciones artísticas como la literatura, pintura, música, etc.

Centrándonos en la relación entre las emociones y las distintas prácticas motrices, Lavega, March y Filella (2013) desarrollaron y validaron, partiendo de la clasificación de emociones de Bisquerra Alzina (2000), una herramienta para el estudio de las emociones que suscitan los juegos deportivos en cada uno de los dominios de acción motriz existentes: psicomotor, de cooperación, de oposición y de cooperación-oposición (Parlebas, 2001). Aplicando esta herramienta en tres estudios distintos, observaron que las relaciones entre emociones y prácticas motrices son muy amplias e intrincadas. Además, éstas presentaban diferencias según cual fuera el dominio de acción bajo el que se encontraba la práctica motriz en cuestión. Si bien, se manifestaban unos índices elevados de emociones positivas para todos los dominios de acción en general, éste y otros trabajos derivados muestran también existencia de las emociones negativas y su influencia según el dominio de acción (Lavega, Aráujo y Jaqueira, 2013; Lavega et al., 2013; Lavega et al., 2014).

Dentro de estas emociones negativas, las que se asocien con acciones motrices que impliquen contacto y, además, dificulten la praxis y/o generen una conducta motriz desajustada, se convertirán en nuestro objeto de estudio: los problemas afectivos derivados del contacto.

A continuación se describirán las principales emociones generadoras de conflicto afectivo en función de la revisión bibliografía realizada mostrada en la Tabla 1.

\subsection{EL MIEDO}

El miedo, ampliamente estudiado en la psicología, representa "una turbación del ánimo, fuerte y espontánea en presencia de un peligro real o imaginario" (Siquier, 1987, p. 26). A su vez, Hongler (1988, p. 21) describe el miedo como "un estado emocional que el sujeto percibe de forma desagradable" y que se muestra cuando:

- "un individuo no es capaz dentro de una situación que él juzga amenazante (estresante)",

- "se suprime la amenaza mediante acciones motrices adecuadas (abandonar el terreno, agredir)",

- "se producen modificaciones de la observación (negar la amenaza) y de las interpretaciones".

De esta forma, este mismo autor afirma, que los fenómenos de estrés y miedo pueden ser identificados en tres niveles diferentes:

- Fisiológico: centrado en aspectos que pueden ser medidos mediante pruebas de laboratorio, como pueden ser las variaciones bioquímicas, de frecuencia cardíaca y respiratoria, entre otras.

- Motor: mediante la existencia de contracciones musculares adyacentes a la realización motriz; por ejemplo, gestos, movimientos parásitos, hipertonicidad, agarrotamiento; o la aparición de comportamientos destinados a modificar o a suprimir el estado de estrés como por ejemplo los balanceos u oscilaciones. 
- Verbal: referido a las expresiones verbales de todos los mecanismos perceptivos, cognitivos y emocionales que pueden ser vividos subjetivamente en una situación.

Dado que, miedo y ansiedad son conceptos próximos, cuyas diferencias vienen determinadas por la presencia o no del estímulo que las genera (Kleinert, 2002; Lavoura, 2007; Lazarus, 2000; Ochoa y De La Puente, 1991); podemos asumir que van a presentar los mismos síntomas. Así, Ochoa y De La Puente (1991) exponen unos parecidos a los planteados por Hongler (1988), diferenciándolos según el sistema funcional:

- Sistema motor: la manifestación más frecuente es la conducta de escape o evitación, por la que el individuo intenta no enfrentarse al estímulo generador de ansiedad. En ocasiones puede manifestarse como un estado de inhibición motriz, donde el individuo queda paralizado.

- Sistema cognitivo: el individuo se siente "preocupado, temeroso, inquieto, con pensamientos de catástrofe, de que «algo va a pasar»", con dificultades de atención y concentración.

- Sistema fisiológico: se produce una sobreactivación del Sistema Nervioso Central como resultado de un estado de alerta mayor. Como consecuencia se incrementa entre otros parámetros, la frecuencia cardíaca, la frecuencia respiratoria y la conductancia de la piel.

Si nos centramos en los aspectos relacionados con el aprendizaje motor, Zubiaur González y Gutiérrez Santiago (2003, p. 26) señalan que el miedo:

... es una emoción que aparece frecuentemente en el aprendizaje motor. Cuyas consecuencias pueden ser perjudiciales, pudiendo desencadenar rechazos, fobias y situaciones estresantes para el aluno. Sin embargo, ha recibido poca atención por parte de los investigadores que se han centrado, casi exclusivamente, en la ansiedad ante la competición.

Si bien para otras emociones hablaremos de forma más general, en el caso del miedo nos circunscribimos a uno específico, el "miedo a la lesión", al ser este, según la bibliografía, el que presenta una vinculación más estrecha con el contacto. El mantenimiento de la integridad física del individuo ha sido estudiada dentro de un amplio abanico de campos. Como veremos en el siguiente apartado, la variedad de puntos de vista hace que exista también una multitud de términos que agrupan esta dimensión de nuestro objeto estudio, siendo los ejemplos más comunes los de "miedo a lesionarse", "miedo a hacerse daño", "miedo a la caída", "miedo al balón".

Ahora bien, no todo el "miedo a lesionarse" tiene relación directa con una acción de contacto con otro individuo, con un objeto o con algún elemento del medio; los casos más claros encontrados en la bibliografía que muestran este aspecto son las denominadas "overuse injury", o "no traumatic injury" (Petersen et al., 2005; Slobounov, 2008, 199; Taylor y Kress, 2006, p. 326) y que se pueden definir como:

An injury was categorized as overuse if no traumatic event could be identified. The classification of an injury as caused with or without contact with another player 
was based on information about the circumstances of the injury given by the player (Petersen et al., 2005, p. 617).

También se han encontrado estudios que se centran en el concepto de "miedo a la caída" tanto en ancianos o en convalecientes de ciertas lesiones, como en diferentes deportes desarrollados en el entorno natural como la escalada (Wuo Pereira, 2010) o el ciclismo de montaña (Lavoura, 2007). También se cita el "miedo a lesionarse" en diferentes deportes que van desde los desarrollados en el medio natural como la escalada y el piragüismo (Lavoura, 2007), hasta los más convencionales como el hockey sobre hielo (Dunn y Nielsen, 1993; Dunn y Causgrove, 2001), la gimnasia deportiva (Chase, Magyar y Drake, 2005) o el fútbol (Dunn y Nielsen, 1993).

Se muestra ahora de una forma más clara la necesidad de estudios sobre las emociones ligadas al contacto en las actividades motrices, tanto en competición como en el aprendizaje, apartado olvidado mayoritariamente por los estudiosos (Zubiaur González y Gutiérrez Santiago, 2003). En este sentido, se ha constatado que el miedo a la lesión es una de las principales causas de abandono de la práctica deportiva en ciertas poblaciones, como son las de mujeres o adultos a partir de cierta edad (Moreno Murcia, Martínez Galindo y Alonso Villodre, 2006; Severinsen, 2011). Incluso puede ser percibido como una barrera para la participación, sobre todo entre la población femenina (Ampong, 2008; Parks, Housemann y Brownson, 2003; Preboth, 2000; Severinsen, 2011; SPARC, 2006 y 2007; Young et al., 2006), llegando a influir incluso en la consecución de una EF escolar realmente coeducativa (Treanor et al., 1998).

Sin embargo, es precisamente la aparición de estas emociones lo que resulta llamativo para ciertos individuos en algunos casos y en determinadas prácticas motrices. Sería, por ejemplo, el caso de la escalada, donde el riesgo se convierte en un aliciente para la participación, como comenta Wuo Pereira (2010, p. 73):

A congruência entre prazer e dor, objetivo e liberdade, constantes na prática, são vividas como partes essenciais do jogo de vertigem, pois ela traz o medo e esse dá prazer. O medo como tempero do movimento de subida é o próprio prato principal, isto é, produz a vontade de arriscar-se e arriscando-se sentimos mais necessidade de sentir o medo novamente. A organização entre prazer e dor e entre medo e coragem são as virtudes vividas na escalada como recompensas.

Por lo tanto, dentro del punto de vista del mantenimiento de la integridad física del individuo, relacionado con el contacto, podemos ver tres dimensiones diferentes según qué o quién se vea involucrado en la acción traumática, y que definiremos a continuación.

\subsubsection{El miedo a la lesión por contacto físico con otro individuo}

Esta primera sub-categoría del miedo a la lesión se centra precisamente en las emociones surgidas de enfrentamientos que entrañan un peligro real e inmediato para la integridad física de un individuo. Una situación motriz típica en la que surgen este tipo de emociones son las acciones cuerpo a cuerpo en rugby.

Éste es el concepto sobre el que más citas bibliográficas se han encontrado (ver Tabla 1), tanto a nivel empírico, fundamentado en la práctica de la docencia de las distintas prácticas 
Estudios Pedagógicos XLVII N 3: 167-190, 2021

LAS EMOCIONES COMO DIFICULTAD DIDÁCTICA EN LA ENSEÑANZA DE PRÁCTICAS MOTRICES: LOS PROBLEMAS AFECTIVOS DERIVADOS DEL CONTACTO. CONCEPTUALIZACIÓN

motrices, como a nivel científico. En torno a él, existe también una gran proliferación terminológica, siendo denominado además de como miedo a la lesión, también como miedo al contacto, miedo al contacto físico, miedo a trabar con el rival, problemas afectivos ligados al contacto; ejemplos éstos de una pequeña muestra de términos traducidos al español.

\subsubsection{El miedo a la lesión por caída: el miedo a caer}

El miedo a caer es otro de los vértices desde el que se pueden estudiar los temores de los deportistas a lesionarse, siendo el suelo el contacto originador, considerado como un elemento de peligro, en función, por ejemplo, de la altura a la que se sitúe la cabeza del individuo, como punto de referencia; o, de la naturaleza del suelo con el que se contacte.

Los estudios que hablan de este tipo de miedo de forma específica se centran, fundamentalmente, en la gimnasia artística deportiva (Cartoni et al., 2001; Cartoni et al., 2002; Chase et al., 2005; Snyder, 1990), si bien es también común encontrar referencias a nivel empírico y didáctico en judo (Roquette, 1994; Santos y Melo, 2003) o rugby, asociado sobre todo a la enseñanza de la acción técnica del placaje (A.R.U., 2011; Collinet, 2000; Damasceno, 2012; Lemaitre, 2003; Rugby Football Union, 1985; Rutherford, 1993; Usero Martín, 1993).

Por último, al igual que en el concepto anterior, la terminología es variada, aunque en menor grado, encontrándose propuestas que van desde visiones genéricas como miedo a lesionarse o problemas afectivos ligados al contacto, hasta opciones concretas como miedo a la caída o temor a hacerse daño al caer (Tabla 1).

\subsubsection{El miedo a la lesión por efecto de un objeto: el miedo al balón}

El último elemento que configura el miedo a la lesión en relación con el contacto es el que está asociado a los temores generados por los objetos que se utilizan en la práctica motriz. El ejemplo que mayoritariamente nos hemos encontrado se refiere al miedo al balón.

El ejemplo más claro de situación motriz generadora de miedo al balón se encuentra referido al rol de portero en balonmano. Tanto es así que algunos autores como Bárcenas y Román (1991) y Silva (2006) consideran fundamental que los jugadores que desempeñen este rol desarrollen un nivel bajo de esta emoción.

En voleibol, Bonnefoy, Lahuppe y Né (2000, p. 96), y Canales y Pina (2014) utilizan el término "miedo al contacto" para describir las reacciones ante situaciones en las que los aprendices evitan encontrarse con los balones para no tener que golpearlos.

\subsection{LA VERGÜENZA}

Para algunos autores esta emoción sería una forma de miedo. Así, por ejemplo, Lavoura y Machado (2007) denominan "medo da exposição". Aquí se ha preferido establecer de forma independiente debido a que presenta una característica peculiar con respecto al contacto que la diferencia del resto de miedos vistos. Esta diferencia se centra en que la vergüenza no compromete la integridad física del individuo ni, por lo tanto, su supervivencia. Más bien, como afirma Lavoura (2007) la vergüenza es "um sentimento (desde que se tenha sua percepção) de desconforto" que en todo caso pudiera "comprometer o relacionamento do indivíduo socialmente e de seu equilíbrio interior". 
En cuanto a la evolución de la vergüenza en el individuo, La Taille (2002, p. 24) la asocia directamente con la consciencia moral del sujeto. Así, afirma que esta emoción pasa de una fase en la que "a vergonha-exposição é a mais forte para outra na qual ela associa-se cada vez mais a valores". Como ejemplo describe como para un niño de hasta seis años el peor de los castigos puede ser quedarse sin recreo, mientras que para uno de mayor edad, a partir de los nueve años, resulta más doloroso un apercibimiento verbal. Se transforma así la percepción del castigo hacia "un decair perante os olhos da pessoa respeitada".

Centrándonos en las prácticas motrices, Espartero Casado y Gutiérrez García (2004, p. 4) señalan desde un punto de vista empírico que, en el caso del judo, "el pudor o el posible sentimiento de vergonzante" generado por el contacto "es uno de los resquemores a los que se puede enfrentar el alumno en este tipo de actividades", dificultándole el aprendizaje. Es más, Grieser et al. (2006) señalan que la vergüenza ("embarrassment") puede ser señalada como barrera para la participación en prácticas motrices en mujeres adolescentes, si bien su incidencia parece ser mucho menor que en el caso del miedo a la lesión.

\subsection{EL ASCO}

Esta emoción hace referencia a la aversión o repugnancia que nos produce una acción, un acontecimiento, un ser o una cosa que nos desagrada profundamente, ya sea por la vivencia de una experiencia real o imaginada. Ahora bien, a pesar de ser considerada por Damasio (2006, p. 179) como una de las "emociones universales básicas", tan solo hemos encontrado una referencia bibliográfica que respalde su inclusión en nuestro objeto de estudio (Salgado López, 2014). Es más, a pesar de basarse en la propuesta de emociones de Bisquerra Alzina (2000), Lavega Burgués et al. (2013) no tienen en cuenta el "asco", como una emoción a investigar al describir las propiedades psicométricas de su herramienta para el estudio de las emociones en prácticas motrices de diferentes dominios práxicos. Sumado a lo anterior, los indicios empíricos, apreciados a lo largo de nuestra carrera profesional en la enseñanza de EF, señalan que esta emoción aparece cotidianamente en nuestras aulas. Así, por ejemplo, es bastante común que los estudiantes la experimenten asco al realizar alguna tarea motriz en el suelo; les toca realizar alguna tarea que implique un contacto con un compañero excesivamente sudoroso o insuficientemente aseado; o, incluso, rozando el racismo, cuando se relacionan individuos de diferentes etnias entre sí, como tristemente observamos cuando en una clase conviven individuos procedentes de exogrupos sociales.

\section{DESCRIPCIÓN DEL CONCEPTO A DEFINIR}

La inexistencia de un término único que defina el concepto relacionado con las dificultades didácticas que surgen de las emociones derivadas del contacto es uno de los mayores problemas al intentar profundizar en su estudio. Tanto es así que hemos encontrado trabajos en los que se cita literalmente: "the term fear of injury is sometimes used but without being defined" (Kleinert, 2002, p. 2). Otro trabajo hacía ver, incluso, que la definición confusa del término modificaba de forma sustancial los resultados entre dos estudios similares sobre ansiedad y riesgo percibido realizados en deportes distintos (Dunn y Syrotuik, 2003).

Creemos que este hecho viene dado por la característica multidimensional del objeto de estudio (Figura 1) presentada en el apartado anterior. 
Figura 1. Representación del carácter multidisciplinar del concepto objeto de estudio

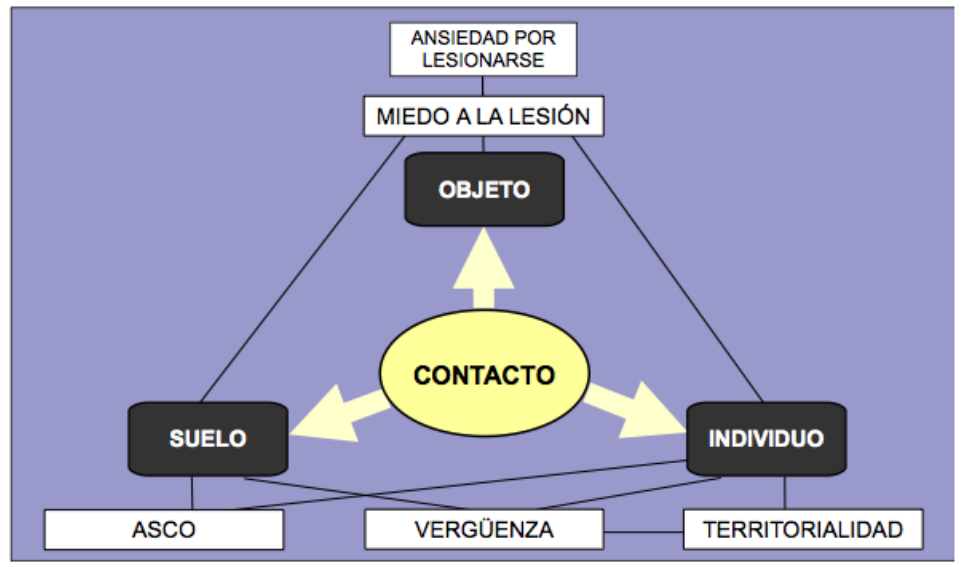

Fuente (elaboración propia).

Por tanto, pocas parecen ser las referencias que abordan los problemas generados por el contacto desde un punto de vista global, tal vez porque son pocos los que lo han estudiado desde un punto de vista didáctico, como elemento distorsionador del aprendizaje.

Bayer (1986, p. 98), es el primer autor que habla de "problemas afectivos" aunque sin delimitar el término. La descripción que realiza se ciñe a decir que podían aparecer en el "comportamiento defensivo de algunos jugadores que rehúsan el contacto, por lo que se apartan o se desplazan sistemáticamente para evitar el balón". Esta falta de conceptualización se ve claramente en la inestabilidad del término, al utilizar también el término "miedo a ser agredido" para describir el concepto anterior en el rol de jugador atacante con balón. Otros autores como Lemaitre (2003) o Usero Martín (1993) también emplean el término "problemas afectivos", perteneciendo al ámbito francófono o influenciados por él.

En general, es muy poca la literatura específica hallada sobre el tema, casi toda de corte empírico y centrándose simplemente en aspectos relacionados con la seguridad de la ejecución a la hora de la enseñanza de las tareas motrices, sin entrar en un análisis más profundo.

Para tener una idea más clara de las dificultades terminológicas asociadas a nuestro objeto de estudio, hemos elaborado un resumen de las principales soluciones adoptadas en cuanto a esta cuestión (Tabla 1). En ella, junto con los autores que utilizan cada uno de los términos, se incluye el aspecto con el que se relaciona y, por último, el tipo de contacto originador del problema afectivo según la categorización de Salgado López et al. (2003). 
Tabla 1. Términos relacionados con el objeto de estudio encontrados en la bibliografía según el año de publicación

\begin{tabular}{|c|c|c|c|}
\hline Autor & Término & Aspecto relacionado & Tipo de contacto \\
\hline $\begin{array}{l}\text { Rugby Football } \\
\text { Union (1985, p. 41) }\end{array}$ & $\begin{array}{l}\text { "fear of physical } \\
\text { contact" } \\
\text { "confidence in the } \\
\text { contact situation" }\end{array}$ & Integridad física & $\begin{array}{c}\text { INTERPERSONALES } \\
\text { BRUSCOS, } \\
\text { CUERPO - SUELO }\end{array}$ \\
\hline Bayer (1986, p. 98) & \begin{tabular}{|l|} 
"problemas afectivos" \\
"miedo a ser agredido"
\end{tabular} & Integridad física & $\begin{array}{c}\text { INTERPERSONALES } \\
\text { BRUSCOS }\end{array}$ \\
\hline $\begin{array}{l}\text { Christina y Corcos } \\
\text { (1988, pp. 58-60) }\end{array}$ & $\begin{array}{l}\text { "fear of injury" } \\
\text { "fear of falling" }\end{array}$ & Integridad física & $\begin{array}{c}\text { INTERPERSONALES } \\
\text { BRUSCOS, } \\
\text { CUERPO - SUELO }\end{array}$ \\
\hline Bilbao (1988) & $\begin{array}{l}\text { "conducta motora } \\
\text { inhibida" }\end{array}$ & Integridad física & CUERPO - SUELO \\
\hline Connes (1988) & $\begin{array}{l}\text { "miedo al contacto" } \\
\text { "problema afectivo" }\end{array}$ & Integridad física & $\begin{array}{l}\text { INTERPERSONALES } \\
\text { BRUSCOS. }\end{array}$ \\
\hline $\begin{array}{l}\text { Feltz (1988, pp. } 154 \\
\text { y } 160)\end{array}$ & \begin{tabular}{|l|} 
"fear of physical pain" \\
"fear of physical harm"
\end{tabular} & Integridad física & CUERPO - SUELO \\
\hline Peys (1991, p. 233) & $\begin{array}{l}\text { "blocages émotionnels } \\
\text { du débutant" }\end{array}$ & Integridad física & $\begin{array}{c}\text { INTERPERSONALES } \\
\text { BRUSCOS }\end{array}$ \\
\hline $\begin{array}{l}\text { Dunn y Nielsen } \\
\text { (1993) }\end{array}$ & $\begin{array}{l}\text { "fear of injury / } \\
\text { physical danger / } \\
\text { physical harm" }\end{array}$ & Integridad física & $\begin{array}{c}\text { INTERPERSONALES } \\
\text { BRUSCOS, } \\
\text { CUERPO - SUELO, } \\
\text { CUERPO - OBJETO }\end{array}$ \\
\hline \multirow{2}{*}{$\begin{array}{l}\text { Rutherford (1993, p. } \\
90 \text { ) }\end{array}$} & "contact" & Integridad física & $\begin{array}{c}\text { INTERPERSONALES } \\
\text { BRUSCOS, } \\
\text { CUERPO - SUELO }\end{array}$ \\
\hline & "confidence in contact" & Integridad física & $\begin{array}{c}\text { INTERPERSONALES } \\
\text { BRUSCOS }\end{array}$ \\
\hline $\begin{array}{l}\text { Usero Martín (1993, } \\
\text { p. 251) }\end{array}$ & $\begin{array}{l}\text { "miedos" o de } \\
\text { "problemas afectivos" }\end{array}$ & Integridad física & $\begin{array}{c}\text { INTERPERSONALES } \\
\text { BRUSCOS, } \\
\text { CUERPO - SUELO, } \\
\text { CUERPO - OBJETO }\end{array}$ \\
\hline Roquette (1994) & $\begin{array}{l}\text { "problemas de medo e } \\
\text { desmotivação" } \\
\text { "medo ou aprensão da } \\
\text { queda" }\end{array}$ & Integridad física & CUERPO - SUELO \\
\hline $\begin{array}{l}\text { Hurrelmann y Klocke } \\
(1997, \text { p. 96) }\end{array}$ & $\begin{array}{l}\text { "fear of physical } \\
\text { contact" }\end{array}$ & Control emocional & $\begin{array}{c}\text { INTERPERSONALES } \\
\text { BRUSCOS, SUAVES }\end{array}$ \\
\hline $\begin{array}{l}\text { Yagüe Cabezón y } \\
\text { Lorenzo Caminero } \\
(1997, \text { p. 26) }\end{array}$ & $\begin{array}{l}\text { "miedo al contacto con } \\
\text { el balón" }\end{array}$ & Integridad física & CUERPO - OBJETO \\
\hline $\begin{array}{l}\text { Heinemann y Puig } \\
\text { (1997) }\end{array}$ & $\begin{array}{l}\text { "aversió pel contacte } \\
\text { corporal" }\end{array}$ & Emociones & INTERPERSONALES \\
\hline
\end{tabular}


Estudios Pedagógicos XLVII N 3: 167-190, 2021

LAS EMOCIONES COMO DIFICULTAD DIDÁCTICA EN LA ENSEÑANZA DE PRÁCTICAS MOTRICES: LOS PROBLEMAS AFECTIVOS DERIVADOS DEL CONTACTO. CONCEPTUALIZACIÓN

\begin{tabular}{|c|c|c|c|}
\hline Autor & Término & Aspecto relacionado & Tipo de contacto \\
\hline $\begin{array}{l}\text { Torres Tobío (1998, } \\
\text { 136) }\end{array}$ & $\begin{array}{l}\text { "temor a la caída"; } \\
\text { "temor a hacerse daño } \\
\text { al caer" }\end{array}$ & Integridad física & CUERPO - SUELO \\
\hline Roffé (1999) & $\begin{array}{l}\text { "miedo a lesionarse y/o } \\
\text { trabar con el rival". }\end{array}$ & Integridad física & $\begin{array}{c}\text { INTERPERSONALES } \\
\text { BRUSCOS }\end{array}$ \\
\hline $\begin{array}{l}\text { Mundina Gómez et } \\
\text { al. (1999, p. } 887)\end{array}$ & $\begin{array}{l}\text { "miedo" / "problema } \\
\text { afectivo" }\end{array}$ & Integridad física & $\begin{array}{l}\text { INTERPERSONALES } \\
\text { BRUSCOS. }\end{array}$ \\
\hline $\begin{array}{l}\text { Bonnefoy et al. } \\
(2000, \text { p. } 96)\end{array}$ & "miedo al contacto" & Integridad física & CUERPO - OBJETO \\
\hline Collinet (2000, p. 13) & $\begin{array}{l}\text { "réticences aux } \\
\text { contacts", "réticences } \\
\text { affectives ou cognitives } \\
\text { au contact" }\end{array}$ & Integridad física & $\begin{array}{c}\text { INTERPERSONALES } \\
\text { BRUSCOS, } \\
\text { CUERPO - SUELO, } \\
\text { CUERPO - OBJETO }\end{array}$ \\
\hline $\begin{array}{l}\text { Heil (2000, pp. 256- } \\
\text { 257) }\end{array}$ & $\begin{array}{l}\text { "fear of reinjury", "fear } \\
\text { of injury" }\end{array}$ & Integridad física & \\
\hline $\begin{array}{l}\text { Ortiz Camacho et al. } \\
(2000, \text { pp. 62-63) }\end{array}$ & "contacto físico" & Comunicación & $\begin{array}{l}\text { INTERPERSONALES } \\
\text { SUAVES }\end{array}$ \\
\hline Preboth (2000) & "fear of injury" & Integridad física & $\begin{array}{c}\text { INTERPERSONALES } \\
\text { BRUSCOS, } \\
\text { CUERPO - OBJETO }\end{array}$ \\
\hline $\begin{array}{l}\text { Fortanasce, Ouelette } \\
\text { y Robinson (2001, p. } \\
55)\end{array}$ & "fear of the ball" & Integridad física & CUERPO - OBJETO \\
\hline Cartoni et al. (2001) & "paura del trauma" & Integridad física & CUERPO - SUELO \\
\hline Teixeira Vaz (2001) & $\begin{array}{l}\text { "problemas no plano } \\
\text { afectivo" }\end{array}$ & Integridad física & $\begin{array}{l}\text { INTERPERSONALES } \\
\text { CUERPO - SUELO }\end{array}$ \\
\hline $\begin{array}{l}\text { Thomas, Launder y } \\
\text { Nelson (2001, p. 47) }\end{array}$ & "fear of injury" & Integridad física & \\
\hline Cartoni et al. (2002) & "paura dell'infortunio" & Integridad física & CUERPO - SUELO \\
\hline $\begin{array}{l}\text { Drauchke (2002, p. } \\
86)\end{array}$ & $\begin{array}{l}\text { "miedo al balón" } \\
\text { "miedo a caerse" }\end{array}$ & Integridad física & $\begin{array}{l}\text { CUERPO - SUELO, } \\
\text { CUERPO - OBJETO }\end{array}$ \\
\hline $\begin{array}{l}\text { Viviani Brochado } \\
\text { (2002) }\end{array}$ & $\begin{array}{l}\text { "medo de se } \\
\text { machucar" } \\
\text { "medo de pasar } \\
\text { vergonha" }\end{array}$ & Integridad física & \\
\hline Kleinert (2002) & $\begin{array}{l}\text { "sport injury trait } \\
\text { anxiety" }\end{array}$ & Integridad física & \\
\hline $\begin{array}{l}\text { Parks et al. (2003, p. } \\
\text { 34) }\end{array}$ & $\begin{array}{l}\text { "fear of injury" } \\
\text { "afraid of injury" }\end{array}$ & Integridad física & \\
\hline $\begin{array}{l}\text { Dunn y Syrotuik } \\
\text { (2003) }\end{array}$ & $\begin{array}{l}\text { "fear of injury or } \\
\text { physical danger" }\end{array}$ & Integridad física & $\begin{array}{c}\text { INTERPERSONALES } \\
\text { BRUSCOS, } \\
\text { CUERPO - SUELO }\end{array}$ \\
\hline
\end{tabular}




\begin{tabular}{|c|c|c|c|}
\hline Autor & Término & Aspecto relacionado & Tipo de contacto \\
\hline $\begin{array}{l}\text { Mendez; Inglés; } \\
\text { Hidalgo; García- } \\
\text { Fernández, y Quiles } \\
\text { (2003) }\end{array}$ & $\begin{array}{l}\text { "miedo al contacto } \\
\text { físico" }\end{array}$ & Comunicación & $\begin{array}{l}\text { INTERPERSONALES } \\
\text { SUAVES }\end{array}$ \\
\hline Lemaitre (2003) & $\begin{array}{l}\text { "problèmes affectifs } \\
\text { liés au contact" }\end{array}$ & Integridad física & $\begin{array}{c}\text { INTERPERSONALES } \\
\text { BRUSCOS, } \\
\text { CUERPO - SUELO }\end{array}$ \\
\hline \multirow{2}{*}{$\begin{array}{l}\text { Zubiaur González y } \\
\text { Gutiérrez Santiago } \\
(2003)\end{array}$} & $\begin{array}{l}\text { "miedo por inseguridad } \\
\text { física" } \\
\text { "miedo a hacerse } \\
\text { daño" }\end{array}$ & Integridad física & $\begin{array}{c}\text { INTERPERSONALES } \\
\text { BRUSCOS, } \\
\text { CUERPO - SUELO, } \\
\text { CUERPO - OBJETO }\end{array}$ \\
\hline & $\begin{array}{l}\text { "miedo a hacer el } \\
\text { ridículo social" }\end{array}$ & Comunicación & INTERPERSONALES \\
\hline Berra (2003a, p. 109) & $\begin{array}{l}\text { "acceptation de } \\
\text { l'anffrontement } \\
\text { physique" }\end{array}$ & \multirow{3}{*}{ Integridad física } & \multirow{3}{*}{$\begin{array}{c}\text { INTERPERSONALES } \\
\text { BRUSCOS, } \\
\text { CUERPO - SUELO }\end{array}$} \\
\hline Berra (2003a, p. 122) & $\begin{array}{l}\text { "appréhension du } \\
\text { contact avec le tapis et } \\
\text { peur de se fair mal" }\end{array}$ & & \\
\hline Berra (2003a, p. 139) & $\begin{array}{l}\text { "appréhension de la } \\
\text { chute non decide car } \\
\text { plus difficile à } \\
\text { anticiper" }\end{array}$ & & \\
\hline Berra (2003b, p. 221) & $\begin{array}{l}\text { "appréhension } \\
\text { physique et/ou } \\
\text { affective" }\end{array}$ & Integridad física & CUERPO - OBJETO \\
\hline $\begin{array}{l}\text { Bischops y Gerards } \\
\text { (2003, p. 29) }\end{array}$ & "fear of the ball" & Integridad física & CUERPO - OBJETO \\
\hline $\begin{array}{l}\text { Ruiz Munuera (2003, } \\
\text { p. 367) }\end{array}$ & $\begin{array}{l}\text { "miedo al contacto } \\
\text { físico" }\end{array}$ & Integridad física & $\begin{array}{c}\text { INTERPERSONALES } \\
\text { BRUSCOS }\end{array}$ \\
\hline $\begin{array}{l}\text { Santos y Melo (2003, } \\
\text { p. 36) }\end{array}$ & $\begin{array}{l}\text { "medo de cair" "receio } \\
\text { de cair" }\end{array}$ & Integridad física & CUERPO - SUELO \\
\hline $\begin{array}{l}\text { Gutiérrez Santiago y } \\
\text { Cancela Carral } \\
\text { (2004) }\end{array}$ & $\begin{array}{l}\text { "miedo a caer" } \\
\text { "miedo a las caídas" }\end{array}$ & Integridad física & CUERPO - SUELO \\
\hline $\begin{array}{l}\text { Short; Reuter; } \\
\text { Brandt; Short, y } \\
\text { Kontos (2004) }\end{array}$ & $\begin{array}{l}\text { "perceived risk of } \\
\text { injury" }\end{array}$ & Integridad física & \\
\hline Cartoni et al. (2005) & $\begin{array}{l}\text { "fear of injury" } \\
\text { "fear of physical } \\
\text { injury" }\end{array}$ & Integridad física & CUERPO - SUELO \\
\hline Mégrot (2005) & $\begin{array}{l}\text { "appréhension du } \\
\text { contact" }\end{array}$ & Integridad física & $\begin{array}{c}\text { INTERPERSONALES } \\
\text { BRUSCOS }\end{array}$ \\
\hline
\end{tabular}


Estudios Pedagógicos XLVII N 3: 167-190, 2021

LAS EMOCIONES COMO DIFICULTAD DIDÁCTICA EN LA ENSEÑANZA DE PRÁCTICAS MOTRICES: LOS PROBLEMAS AFECTIVOS DERIVADOS DEL CONTACTO. CONCEPTUALIZACIÓN

\begin{tabular}{|c|c|c|c|}
\hline Autor & Término & Aspecto relacionado & Tipo de contacto \\
\hline Chase et al. (2005) & "fear of injury" & Integridad física & CUERPO - SUELO \\
\hline Cogan $(2006,654)$ & "fear of injury" & Integridad física & CUERPO - SUELO \\
\hline $\begin{array}{l}\text { Collinet y Nérin } \\
(2006, \text { p. 49). }\end{array}$ & "miedo a tacklear" & Integridad física & $\begin{array}{c}\text { INTERPERSONALES } \\
\text { BRUSCOS }\end{array}$ \\
\hline $\begin{array}{l}\text { Hanson (2006, p. } \\
163)\end{array}$ & $\begin{array}{l}\text { "fear of physical } \\
\text { injury" }\end{array}$ & Integridad física & $\begin{array}{c}\text { (especialmente) } \\
\text { CUERPO - OBJETO }\end{array}$ \\
\hline $\begin{array}{l}\text { Monsma y Feltz } \\
(2006, \text { p. 442) }\end{array}$ & $\begin{array}{l}\text { "fear of falling or } \\
\text { injuring a partner" }\end{array}$ & Integridad física & CUERPO - SUELO \\
\hline $\begin{array}{l}\text { Pérez Turpin y Suárez } \\
\text { Llorca }(2006, \text { p. } 83)\end{array}$ & "miedo al contacto" & Integridad física & $\begin{array}{c}\text { INTERPERSONALES } \\
\text { BRUSCOS }\end{array}$ \\
\hline Silva (2006, p. 227) & "fear of the ball" & Integridad física & CUERPO - OBJETO \\
\hline $\begin{array}{l}\text { Taylor y Kress (2006, } \\
\text { pp. } 336 \text { y } 337)\end{array}$ & $\begin{array}{l}\text { "fear of physical harm" } \\
\text { "fear of bodily injury" }\end{array}$ & Integridad física & \\
\hline Young et al. (2006) & "fear of injury" & Integridad física & \\
\hline Rumin (2006, p. 37) & "miedo al contacto" & Integridad física & $\begin{array}{c}\text { INTERPERSONALES } \\
\text { BRUSCOS }\end{array}$ \\
\hline Chapple (2007) & "fear of the ball" & Integridad física & CUERPO - OBJETO \\
\hline $\begin{array}{l}\text { Griffey y Housner } \\
(2007, \text { p. 46) }\end{array}$ & "fear of injury" & Integridad física & CUERPO - OBJETO \\
\hline Lavoura (2007) & $\begin{array}{l}\text { "medo de cair" } \\
\text { "medo de se } \\
\text { machucar" } \\
\text { "medo da lesão" }\end{array}$ & Integridad física & $\begin{array}{c}\text { INTERPERSONALES } \\
\text { BRUSCOS, } \\
\text { CUERPO - SUELO }\end{array}$ \\
\hline $\begin{array}{l}\text { Peitersen (2007, p. } \\
\text { 59) }\end{array}$ & "miedo al balón" & Integridad física & CUERPO - OBJETO \\
\hline Slobounov (2008) & $\begin{array}{l}\text { "fear of injury" } \\
\text { "fear of re-injury" }\end{array}$ & $\begin{array}{l}\text { Recuperación de } \\
\text { lesiones }\end{array}$ & \\
\hline $\begin{array}{l}\text { Troya Montañez y } \\
\text { Cuéllar Moreno } \\
(2008)\end{array}$ & $\begin{array}{l}\text { "miedo al contacto con } \\
\text { los otros" }\end{array}$ & $\begin{array}{l}\text { Expresión y } \\
\text { Comunicación }\end{array}$ & $\begin{array}{l}\text { INTERPERSONALES } \\
\text { SUAVES }\end{array}$ \\
\hline $\begin{array}{l}\text { Lieberman y } \\
\text { Houston-Wilson } \\
(2009, \text { p. 63) }\end{array}$ & "fear of injury" & Integridad física & CUERPO - OBJETO \\
\hline $\begin{array}{l}\text { Heinen, Pizzera y } \\
\text { Cottyn (2009) }\end{array}$ & "fear of injury" & Integridad física & CUERPO - SUELO \\
\hline $\begin{array}{l}\text { Doctor, Adamec y } \\
\text { Kahn (2010, pp. } 61 \text { e } \\
88)\end{array}$ & $\begin{array}{l}\text { "aphenphobia" } \\
\text { "fear of beaing } \\
\text { touched" } \\
\text { "fear of physical } \\
\text { contact" } \\
\text { "haphephobia" }\end{array}$ & Fobia social & $\begin{array}{l}\text { INTERPERSONALES } \\
\text { SUAVES }\end{array}$ \\
\hline
\end{tabular}




\begin{tabular}{|c|c|c|c|}
\hline Autor & Término & Aspecto relacionado & Tipo de contacto \\
\hline $\begin{array}{l}\text { Walker, Thatcher y } \\
\text { Lavallee (2010) }\end{array}$ & "re-injury anxiety" & Integridad física & \\
\hline A.R.U. (2011) & "confidence in contact" & Integridad física & $\begin{array}{c}\text { INTERPERSONALES } \\
\text { BRUSCOS } \\
\text { CUERPO - SUELO }\end{array}$ \\
\hline $\begin{array}{l}\text { Coqueiro Pires de } \\
\text { Sousa y Caramaschi } \\
(2011, \text { p. 625) }\end{array}$ & $\begin{array}{l}\text { "aversão ao contato } \\
\text { corporal" }\end{array}$ & Comunicación & $\begin{array}{c}\text { INTERPERSONALES } \\
\text { SUAVES }\end{array}$ \\
\hline Turrán Eguren (2011) & $\begin{array}{l}\text { "problemas psico- } \\
\text { afectivos derivados del } \\
\text { contacto cporal" }\end{array}$ & Comunicación & $\begin{array}{l}\text { INTERPERSONALES } \\
\text { SUAVES }\end{array}$ \\
\hline $\begin{array}{l}\text { Aguilar Aguilar } \\
\text { (2012) }\end{array}$ & "miedo al balón" & Integridad física & CUERPO - OBJETO \\
\hline $\begin{array}{l}\text { Canales y Pina } \\
(2014)\end{array}$ & "miedo al contacto" & Integridad física & $\begin{array}{c}\text { INTERPERSONALES } \\
\text { BRUSCOS } \\
\text { CUERPO - OBJETO }\end{array}$ \\
\hline $\begin{array}{l}\text { Salgado López } \\
\text { (2014) }\end{array}$ & $\begin{array}{l}\text { "problemas afectivos } \\
\text { derivados del contacto" }\end{array}$ & $\begin{array}{l}\text { Integridad física } \\
\text { Comunicación }\end{array}$ & $\begin{array}{c}\text { INTERPERSONALES } \\
\text { BRUSCOS Y SUAVES } \\
\text { CUERPO - OBJETO } \\
\text { CUERPO - SUELO }\end{array}$ \\
\hline $\begin{array}{l}\text { Canales y Casedas } \\
(2018)\end{array}$ & "miedo al contacto" & Integridad física & $\begin{array}{c}\text { INTERPERSONALES } \\
\text { BRUSCOS } \\
\text { CUERPO - OBJETO } \\
\text { CUERPO - SUELO }\end{array}$ \\
\hline
\end{tabular}

Fuente. Elaboración propia.

En la Tabla 1 se puede apreciar al existencia de términos idénticos para identificar conceptos distintos o viceversa. Por ejemplo miedo al contacto físico aparece relacionado con un aspecto comunicativo o social del contacto y con el mantenimiento de la integridad física del individuo; en francés, problèmes affectifs liés au contact referido a las colisiones entre sujetos como con el suelo; y en este mismo sentido, fear of injury, en inglés. Es, por tanto, necesario detenernos en la conceptualización del mismo.

Para nosotros, el concepto que agrupa las dificultades didácticas asociadas con la afectividad derivada del contacto agrupa todas las visiones recogidas en la Tabla 1. De esta forma, está relacionado con una reacción emocional negativa que influye en la conducta motriz de un sujeto, ya sea al poner en compromiso su integridad física (como puede ser en el caso de un lanzamiento en caída en balonmano), o bien, al entrar en juego aspectos culturales, como es el caso de los contactos socialmente no admitidos en determinadas zonas del cuerpo (como se da en el agarre de segunda, también en rugby).

Si nos centramos en el mantenimiento de la integridad física, la cuestión se complica más, ya que las distintas fuentes del contacto desencadenarán "problemas afectivos" distintos. Esta visión es la propuesta por Collinet (2000) y Usero Martín (1993), centrándose únicamente en el miedo a colisionar con otro individuo, miedo al balón o miedo a la caída. 
Estudios Pedagógicos XLVII Nº 3: 167-190, 2021

LAS EMOCIONES COMO DIFICULTAD DIDÁCTICA EN LA ENSEÑANZA DE PRÁCTICAS MOTRICES: LOS PROBLEMAS AFECTIVOS DERIVADOS DEL CONTACTO. CONCEPTUALIZACIÓN

Relacionando estos tres tipos de contactos generadores con las dimensiones del objeto de estudio mostradas en la Figura 1, obtenemos una visión completa del concepto al que nos estamos refiriendo (Figura 2). Así, los contactos entre individuos podrían generar problemas en función del mantenimiento de la integridad física, tal como acabamos de ver, pero también desde el punto de vista de aspectos relacionados con la territorialidad o cuestiones psico-morales, como la vergüenza y/o el asco. Por su parte, el suelo tendría relación con aspectos relacionados con el mantenimiento de la integridad física y aspectos psico-morales, ya que la territorialidad se centra en el uso del espacio entre individuos. Por último, el contacto con objetos sólo parece relacionarse con el mantenimiento de la integridad física.

Figura 2. Representación conceptual de los problemas afectivos derivados del contacto.

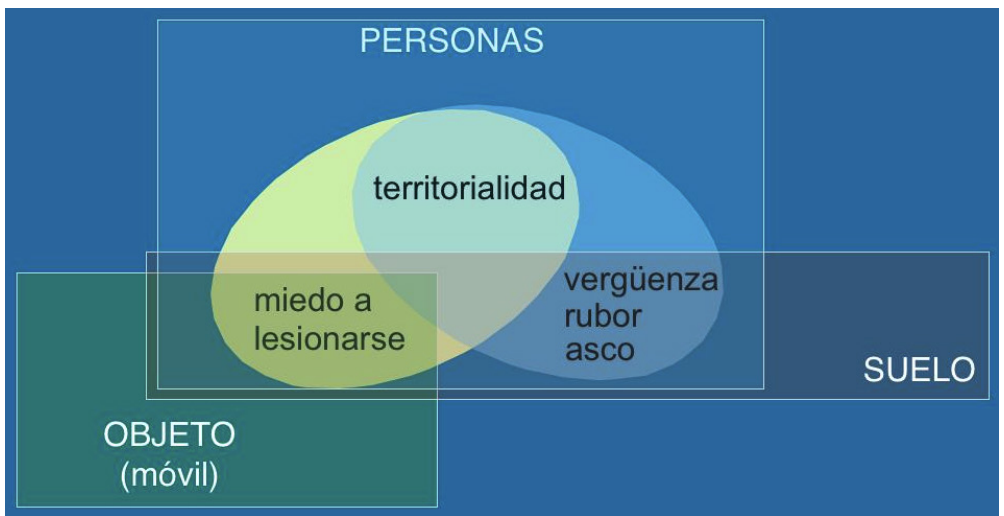

Fuente (elaboración propia).

\subsection{PROPUESTA TERMINOLÓGICA JUSTIFICADA}

Aunque el título del presente trabajo aparece ya decantado hacia una opción terminológica al citar los problemas afectivos derivados del contacto, parece necesario justificar esta decisión antes definir el concepto.

No parece correcto referirse al concepto objeto de este estudio con el término aislado de contacto, aunque son comunes expresiones como: "el contacto no constituye un gran problema que haya que resolver", "aceptar e integrar el aspecto «contacto» del juego" (Collinet y Nerin, 2006, pp. 50, 61) o "grado de aceptación al contacto" (Rumin, 2006, p. 75). Así, el uso de este término puede resultar confuso ya que sólo describe "la acción de tocar" sin quedar asociada a una emoción determinada (miedo, vergüenza y/o asco).

Se debe tener en cuenta también que ciertos tipos de contacto producidos en algunas actividades no tienen por qué ser generadores de ansiedad. Es el caso, por ejemplo de actividades como andar o correr. Por esta razón, para delimitar un poco más el concepto, en nuestra propuesta terminológica debemos añadir una referencia a la turbación del ánimo generada en un individuo cuando, bajo cierta circunstancias, toca a otra persona, objeto o 
incluso el mismo suelo. O lo que es lo mismo, debemos introducir un término que haga referencia al problema, miedo, ansiedad o aversión que producen esos contactos.

Centrándonos en nuestra propuesta terminológica, los términos miedo o aversión implican en sí mismos cierto aspecto de graduación, por lo que se hace necesario un vocablo que los agrupe. Además de esto, el uso término ansiedad tampoco es una opción acertada ya que se diferencia del miedo por la presencia o no del estímulo que las genera (Kleinert, 2002; Lavoura, 2007; Lazarus, 2000; Ochoa y De La Puente, 1991). Por lo tanto, aunque, como dijimos, el término mayoritario a nivel empírico es miedo al contacto, pensamos que debe establecerse un término que supere la confusión existente dentro de este. Ello es debido a:

- El término miedo al contacto no distingue las diferentes dimensiones existentes dentro del concepto presentado; es decir, no queda claro si se refiere únicamente a aspectos relacionados con la invasión del espacio personal; de mantenimiento de la integridad física; a las reacciones de rechazo por considerar sucio el objeto a contactar, o; si integra a todas.

- Tradicionalmente, el término miedo al contacto ha dejado fuera las emociones surgidas de otras fuentes de dificultades didácticas relacionas con el contacto, como son el espacio (suelo) y los objetos (como por ejemplo el móvil).

- Por último, el término miedo al contacto eliminaba también, la posibilidad de su gradación, como hemos dicho al hablar de la distinción entre miedo y aversión.

Analizada la bibliografía, hemos optado por utilizar el término propuesto por la corriente francesa, es decir: problemas afectivos. Esta decisión viene apoyada también por nuestra visión didáctica del concepto, marcando de esta forma la influencia negativa para la optimización del aprendizaje motor en el caso de aparecer estas emociones. Esto diferencia el concepto objeto de estudio de las emociones que lo generan (miedo, vergüenza, asco) que, como hemos visto, no pueden ser consideradas en sí mismas como negativas (Bisquerra Alzina, 2000 y 2003; Damasio, 2006; La Taille, 2002; Lazarus, 2000).

En cuanto a las decisiones tomadas para incluir el término contacto, somos conscientes de la existencia de otras expresiones posibles y conocidas en la literatura, como pueden ser miedo a lesionarse, miedo al balón, miedo a la caída, entre otras. De hecho, la tendencia de separar los distintos problemas afectivos en diferentes términos, se da incluso dentro de un mismo autor. Así, por ejemplo, Viviani Brochado (2002, p. 71) asocia el concepto de "medo de se machucar" con la gimnasia artística deportiva y el de "medo de pasar vergonha" con la danza. Bajo nuestro punto de vista, todos ellos representan parcialmente el objeto de estudio y, en todo caso, describen distintas dimensiones del mismo. En lo que se refiere al uso de la parte específica de la expresión, hemos encontrado también cierta diferenciación, variando, según los autores, entre tacto y contacto. Para la toma de decisiones en este sentido se consultaron las distintas acepciones recogidas para ambos términos en los diccionarios de uso habitual de las lenguas en las que se realizó la búsqueda bibliográfica: gallego, español, portugués, inglés y francés.

Tradicionalmente parece que la literatura en español sobre el contacto ha empleado, mayoritariamente, el término tacto para traducir el inglés touch. Ahora bien, las acepciones de tacto en gallego-portugués y español (Associaçom Galega da Lingua, 2019; Porto Editora, n. d.; Real Academia Española, 2001; Real Academia Galega, 2012), son 
claramente diferentes de las del inglés touch (Oxford Dictionaries, n. d.). Así, la primera se refiere expresamente al sentido que recoge informaciones surgidas cuando un individuo toca a otro, a un objeto o a cualquier elemento externo a él. Por el contrario, en inglés el significado principal del término touch corresponde con los significados principales del término contacto en los otros idiomas. En todo caso, la utilización de tacto en ese sentido representaría una segunda acepción en español y francés (Larousse, n. d.).

Visto lo anterior, se debe considerar que el término de uso correcto, dentro de nuestro objeto de estudio para las lenguas romances analizadas, debería ser contacto.

El introducir el vocablo derivados, además de estar respaldado por la bibliografía de la corriente francesa antes citada, no deja de ser sino una necesidad sintáctica para dar sentido completo a la expresión frente a un posible problemas afectivos del contacto, que suena totalmente extraño en nuestro idioma.

La última justificación que resta se refiere a la supresión de ciertos calificativos que algunos autores introducían al referirse al contacto como físico (Méndez et al., 2003; Ortiz Camacho et al., 2000), physical en inglés (Doctor, Adamec y Kahn, 2010; Wehmeier, 2000), o corporal (Lagardera Otero, 1999). Para nosotros es redundante en todos los casos, ya que todo contacto se realiza en el mundo físico y con el cuerpo del individuo implicado en la acción. Tal diferenciación representa una visión dual del individuo, fruto de la tradición cultural occidental. Además, esta reiteración convierte el término en reduccionista, solo refiere un tipo de contacto, los que se dan entre individuos, no abarcando todos los contactos posibles en las prácticas motrices.

\section{CONCLUSIÓN: DEFINICIÓN DEL CONCEPTO PROBLEMAS AFECTIVOS DERIVADOS DEL CONTACTO}

A lo largo de este trabajo se ha mostrado la necesidad de conceptualizar de forma unitaria los aspectos referidos a las dificultades didácticas que surgen de las emociones derivadas del contacto en los procesos de enseñanza de prácticas motrices. En este sentido, se ha presentado una visión conceptual que integra tanto las dificultades didácticas surgidas de emociones ligadas a contactos comprometedores para la integridad física del individuo, asociadas al miedo, como las surgidas de emociones como la vergüenza o el asco, asociadas a aspectos socio-culturales. Se ha enfatizado, en todo caso, que se trata de un concepto con matiz negativo desde la perspectiva didáctica, ya que interfiere en el proceso de aprendizaje motor, limitando su optimización. En este sentido, como conclusión del trabajo, podemos definir el concepto problema afectivo derivado del contacto como toda dificultad de orden didáctico, que influye negativamente en el aprendizaje motor de un individuo, debido a una reacción emocional asociada a una acción de contacto con otros individuos, objetos o suelo, que interfiere en la toma de decisión y puede dar, como resultado, una conducta motriz desajustada.

Junto con lo anterior, se ha justificado la opción terminológica expuesta tanto desde un punto de vista del léxico español, como en lo que se refiere a las connotaciones negativas para el aprendizaje antes citadas.

Por último, como líneas de trabajo futuras, sería necesario concretar qué tipo de tareas motrices son generadoras de problemas afectivos para que el docente pueda gestionarlas de manera correcta en sus secuencias de aprendizaje. En este sentido, convendría también 
definir científicamente, cuáles de los parámetros para modular este tipo de problemas mostrados en la bibliografía son los fundamentales en cada caso. Esto facilitaría al docente una herramienta para afrontar la enseñanza de las prácticas motrices que impliquen contacto.

\section{REFERENCIAS BIBLIOGRÁFICAS}

A.R.U. (2011). Smart Rugby: Confidence in Contact. Sidney: Australian Rugby Union. http://www. rugbymadrid.com/index.php?seccion=direccion_tecnica

Aguilar Aguilar, R. D. (2012). Curso básico de balonmano. http://viref.udea.edu.co/

Ampong, J. (2008). Factors associated with low participation of females in university sports in Ghana. Dissertation of Master of Philosophy Degree in Physical Education. University of Cape Coast. http://ir.ucc.edu.gh/dspace/browse-title?top=123456789\%2F1525

Associaçom Galega da Lingua (2019). Dicionário Estraviz. http://www.estraviz.org/

Bárcenas, D. y Román, J. de D. (1991). Balonmano. Técnica y Metodología. Madrid: Gymnos.

Bayer, C. (1986). La Enseñanza de los Juegos Deportivos Colectivos. Barcelona: Hispano-Europea. Bernard, M. (1985). El cuerpo. Barcelona: Paidós Ibérica.

Berra, C. (2003a). Lutte. En P. Seners (Eds.), Conduire ou maîtriser un affrontement individuel ou collectif (pp. 105-218). Paris: Vigot.

. (2003b). Volley-ball. En P. Seners (Eds.), Conduire ou maittriser un affrontement individuel ou collectif (pp. 219-319). Paris: Vigot.

Bilbao, A. (1988). Efecto de una técnica conductual en el tratamiento de conductas motoras inhibidas. Motricidad, (2), 105-114.

Bischops, K. y Gerards, H-W. (2003). Soccer Training for Girls. Oxford: Meyer \& Meyer Verlag.

Bisquerra Alzina, R. (2000). Educación emocional y bienestar. Barcelona: Praxis. . (2003). Educación emocional y competencias básicas para la vida. Revista de Investigación Educativa, 21(1), 7-43. http://revistas.um.es/rie/article/view/99071/94661

Bonnefoy, G., Lahuppe, H. y Né, R. (2000). Enseñar voleibol para jugar en equipo. Barcelona: INDE.

Canales, I. (2006). Consecuencias pedagógicas de la mirada y el tacto en la expresión corporal. Tesis doctoral. I.N.E.F.C. Lleida. Catalunya. http://hdl.handle.net/10803/8205

Canales, I. y Pina-Blanco, I. (2014). El miedo al contacto en el voleibol. percepciones del alumnado de educación física. Agora para la educación física y el deporte, 16(2), 122-136. http://agorarevista.blogs.uva.es/files/2014/12/agora_16_2c_canales_et_pina.pdf

Canales, I. y Casedas, A. (2018). Miedo al Contacto en BalonmanoPercibido por los Jugadores de unEquipo de Categoría Alevín. Kronos, 17(2), 1-11. https://abacus.universidadeuropea.es/ bitstream/handle/11268/7776/Kronos_2018_17_2_1.pdf;jsessionid=4C640F45F66C26CDAB C6290F8B86CD82? sequence $=2$

Cartoni, A. C., Damassa, G., De Pero, R. y Minganti C. (2001). Confronto di genere e paura nella ginnastica artistica. Movimento, 17(2), 27-30.

Cartoni, A. C., Massaro, A., Minganti, C. y Zelli, A. (2002). La paura dell'infortunio in ginnasti di alto livello. SDS: Rivista di Cultura Sportiva, 21(56), 19-26.

Cartoni, A., Minganti, C. y Zelli, A. (2005). Gender, age, and professional-level differences in the psychological correlates of fear of injury in italian gymnasts. Journal of Sport Behavior, $28(1), 3$.

Chapple, A. (2007). Coaching Volleyball: Top 5 ways to use 'pepper' to improve your game. e-sports. http://www.e-sports.com/articles/1771/1/Coaching-Volleyball-Top-5-ways-to-use-pepper-toimprove-your-game/Page1.html 
Estudios Pedagógicos XLVII N 3: 167-190, 2021

LAS EMOCIONES COMO DIFICULTAD DIDÁCTICA EN LA ENSEÑANZA DE PRÁCTICAS MOTRICES: LOS PROBLEMAS AFECTIVOS DERIVADOS DEL CONTACTO. CONCEPTUALIZACIÓN

Chase, M. A., Magyar, T. M. y Drake, B. M. (2005). Fear of injury in gymnastics: Self-efficacy and psychological strategies to keep on tumbling. Journal of Sports Sciences, 23(5), 465-475.

Christina, R. W. y Corcos, D. M. (1988). Coaches guide to teaching sports skills. Champaign, Illinois: Human Kinetics.

Cogan, K. D. (2006). Sport Psychology in Gymnastics. En J. Dosil (Eds), The Sport Psychologist's Handbook: A guide for sport-specific performance enhancement. (pp. 641-664). West Sussex: John Wiley \& Sons Ltd.

Collinet, S. (2000). Les Fondamentaux du Rugby: Manuel pour l'éducateur et l'entraîneur. Niveau 1. Niveau 2. Paris: Anphora.

Collinet, S. y Nérin, J-Y. (2006). Rugby. ¿Cómo enseñar el deporte hoy? De la escuela al club. Buenos Aires: Stadium.

Connes, M. (1988). Pedagogía moderna del rugby. Revista de Educación Física, (23), 31-33.

Damasceno, N. (2012). Quaderno de exercícios. Rugby. Associação de Rugby do Norte / Federação Portuguesa de Rugby. http://www.fpr.pt/FICHEIROS_SITE_FPR/documentos/ desenvolvimento/CadernoExercicios_2012_WEB.pdf

Damasio, A. R. (2006). El error de Descartes. La emoción, la razón y el cerebro humano. Barcelona: Crítica.

Davis, F. (1987). La comunicación no verbal. Madrid: Alianza Editorial.

Descamps, M-A. (1990). El lenguaje del cuerpo y la comunicación corporal. Bilbao: Deusto.

Doctor, R. M., Adamec, C. A. y Kahn, A. P. (2010). The Encyclopedia of Phobias, Fears, and Anxieties. New York: Facts on File.

Drauchke, K. (2002). El entrenador de voleibol. Barcelona: Paidotribo.

Dunn, J. G. H. y Causgrove Dunn, J. (2001). Relationships among the Sport Competition Anxiety Test, the Sport Anxiety Scale, and the Collegiate Hockey Worry Scale. Journal of Applied Sport Psychology, (13), 411-429.

Dunn, J. G. H. y Nielsen, A. B. (1993). A between-sport comparison of situational threat perceptions in ice hockey and soccer. Journal of Sport \& Exercise Psychology, (15), 449-465.

Dunn, J. G. H. y Syrotuik, D. G. (2003). An investigation of multidimensional worry dispositions in a high contact sport. Psychology of Sport and Exercise, 4(3), 265-282.

Espartero Casado, J. y Gutiérrez García, C. (2004). El judo y las actividades de lucha en el marco de la Educación Física Escolar: una revisión de las propuestas y modelos de su enseñanza. Actas al III Congreso de la Asociación Española de Ciencias del Deporte. Valencia. http:// cienciadeporte.eweb.unex.es/congreso/04\%20val/pdf/0 eljudo.pdf

Fast, J. (1994). El sublenguaje del cuerpo: Gestos, posturas y distancias en las relaciones personales en la empresa. Barcelona: Paidós.

Feltz, D. L. (1988). Gender differences in the causal elements of self-efficacy on a high avoidance motor Task. Journal of Sport \& Exercise Psychology, (10), 151-166.

Fortanasce, V., Ouelette, J. y Robinson, L. J. (2001). The official American youth soccer organization handbook. New York: American Youth Soccer Organization Simon and Schuster.

Grieser, M, Vu, M. B., Bedimo-Rung, A. L., Neumark-Sztainer, D., Moody, J., Young, D. R. y Moe, S. G. (2006). Physical Activity Attitudes, Preferences, and Practices in African American, Hispanic, and Caucasian Girls. Health Education \& Behavior, 33(1), 40-51. www.ncbi.nlm.nih. gov/pmc/articles/PMC2494874/pdf/nihms52949.pdf

Griffey, D. C. y Housner L. D. (2007). Designing effective instructional tasks for physical education and sports. Champaign. Illinois: Human Kinetics.

Gutiérrez Santiago, A. y Cancela Carral, J. M. (2004). Una propuesta de aprendizaje del juego acrobático en el voleibol. Lecturas: educación física y deportes, (74). http://www.efdeportes. com/efd74/voleib.htm

Hall, E. T. (1989). El lenguaje silencioso. Madrid: Alianza Editorial. . (2003). La dimensión oculta. Madrid: Siglo XXI. 
Hanson, T. (2006). Focused baseball: using sport psychology to improve baseball performance. En J. Dosil (Eds.), The Sport Psychologist's Handbook: A Guide for Sport-Specific Performance Enhancement (pp. 159-182). West Sussex: John Wiley \& Sons Ltd.

Heil, J. (2000). The injured athlete. En Y. Hanin (Ed.), Emotions in Sport (pp. 245-265). Champaign, Illinois: Human Kinetics.

Heinemann, K. y Puig, N. (1997). Emocions en l'esport. Esbós d'una anàlisi sociològica. Revista Catalana de Sociología, (5), 117-146. http://www.raco.cat/index.php/revistasociologia/article/ view Article/236218/0

Heinen, T.; Pizzera, A. y Cottyn, J. (2009). When is manual guidance effective for the acquisition of complex skills in Gymnastics?. International Journal of Sport Psychology, (40), 1-22.

Hernández Moreno, J. (1994). Análisis de las estructuras del juego deportivo. Barcelona: INDE.

Hongler, R. (1988). El stress y el miedo en el deporte. Revista de Entrenamiento Deportivo, 2(4), 20-31.

Hurrelmann, K. y Klocke, A. (1997). The role of the school in comprehensive health promotion. En R. Takanishi \& D. A. Hamburg, Preparing Adolescents for the Twenty-First Century: Challenges Facing Europe and the United States (pp. 82-107). Cambridge: Cambridge University Press.

Kleinert, J. (2002). An approach to sport injury trait anxiety. European Journal of Sport Science, 2(3), 1-12. http://journals.humankinetics.com/AcuCustom/Sitename/Documents/DocumentItem/137. pdf

La Taille, Y. (2002). O sentimento de vergonha e suas relações com a moralidade. Psicologia: Reflexão e Crítica, 15(1), 13-25. http://www.scielo.br/pdf/prc/v15n1/a03v15n1.pdf

Lagardera Otero, F. (dir.) (1999). Diccionario Paidotribo de la Actividad Física y el Deporte. Barcelona: Paidotribo.

Larousse (n.d.). Dictionnaires de Français. http://www.larousse.fr/dictionnaires/francais

Lavega, P., March, J. y Filella, G. (2013). Juegos deportivos y emociones. Propiedades psicométricas de la escala GES para ser aplicada en la Educación Física y el Deporte. Revista de Investigación Educativa, 31(1), 151-165. http://dx.doi.org/10.6018/rie.31.1.147821

Lavega, P., Filella, G., Lagardera, F., Mateu, M. y Ochoa, J. (2014). Juegos motores y emociones. Cultura y educación, 25(3), 347-360. https://doi.org/10.1174/113564013807749731

Lavega, P., Aráujo, P. y Jaqueira, A. R. (2013c). Teaching motor and emotional competencies in university students. Cutura_Ciencia_Deporte, 8(22), 5-15. http://ccd.ucam.edu/index.php/ revista/article/view/219/204

Lavoura, T. N. (2007). Estados Emocionais: A Investigação do medo no contexto esportivo. Tese de Mestrado. Universidade Estadual Paulista. Brasil. http://www.educadores.diaadia.pr.gov.br/ arquivos/ File/2010/artigos_teses/EDUCACAO_FISICA/dissertacao/BoletimEF.org_Estadosemocionais-a-investigacao-do-medo-no-contexto-esportivo.pdf,

Lavoura, T. N. y Machado, A. A. (2007). A vergonha e sua relação com a prática esportiva: um estudo de caso. Motriz, 13(1), 64-71. http://www.lepespe.com.br/downloads/1_-_A_vergonha_e_sua_ relacao_com_a_pratica_esportiva_um_estudo_de_caso.pdf

Lazarus, R. S. (2000). How emotions influence performance in competitive sports. The Sport Psychologist, (14), 229-252. http://tonypickering.com/documents/Lazarus_2000.pdf

LeDoux, J. (2012). Rethinking the emotional brain. Neuron, 73(4), 653-676. https://www. researchgate.net/publication/221861844_Rethinking_the_emotional_brain?ev=prf_pub

Lemaitre, Ph. (2003). Avancer: Résolution des problèmes affectifs liés au contact. EPS, (303), 35-38.

Lieberman, L. J. y Houston-Wilson, C. (2009). Strategies for Inclusion: A Handbook for Physical Educators. Champaign. Illinois: Human Kinetics.

Martínez-Martínez, F. D. y Valero-Valenzuela, A. (2019). Vivencia emocional del alumnado universitario en educación física: revisión sistemática. Revista Iberoamericana de psicología del ejercicio y el deporte, 14(2), 91-96. https://www.riped-online.com/articles/emotionalexperience-of-university-students-in-physical-education-systematic-review.pdf 
Estudios Pedagógicos XLVII N 3: 167-190, 2021

LAS EMOCIONES COMO DIFICULTAD DIDÁCTICA EN LA ENSEÑANZA DE PRÁCTICAS MOTRICES: LOS PROBLEMAS AFECTIVOS DERIVADOS DEL CONTACTO. CONCEPTUALIZACIÓN

Mégrot, F. (2005). Etude de la génération de trajectoires locomotrices et de la distance chez les combattants: Escrime, Judo. Paris. INSEP. Département des Sciences du Sport, Laboratoire Mouvement, Action et Performance. http://www.sportdocs.insep.fr/flora_insep/jsp/index_view_ direct_anonymous.jsp?record=aoinsep:DC_ARCHI:458

Mendez, F. X., Inglés, C. J., Hidalgo, Ma. D., García-Fernández, J. M. y Quiles, Mª. J. (2003). Los miedos en la infacia y la adolescencia: un estudio descriptivo. Revista Electrónica de Motivación y Emoción, 6(13), 1-17. http://reme.uji.es/articulos/amxndf4650710102/texto.html

Monsma, E. V. y Feltz, D. (2006). A Mental Preparation Guide for Figure Skaters: A Developmental Approach. En J. Dosil (Eds.), The Sport Psychologist's Handbook: A Guide for Sport-Specific Performance Enhancement, (pp. 427-454). West Sussex: John Wiley \& Sons Ltd.

Montagu, A. y Matson, F. (1989). El contacto humano. México: Paidós Studio.

Moreno Murcia, J. A, Martínez Galindo, C. y Alonso Villodre, N. (2006). Actitudes hacia la práctica físico-deportiva según el sexo del practicante. Revista Internacional de Ciencias del Deporte. 3(2), 20-43. http://www.cafyd.com/REVISTA/art2n3a06.pdf

Mujica-Johnson, F. N., Orellana-Arduiz, N. C. y Concha-López, R-F. (2017). Emociones en la clase de Educación Física: revisión narrativa (2010-2016). Ágora para la Educación Física y el Deporte 19(1), 119-134. 10.24197/aefd.1.2017.119-134

Mujica-Johnson, F. N. y Salgado López, J. I. (2021). Cultura, emociones y aprendizaje significativo en Educación Física. Revista Ensayos Pedagógicos, 16(2), 63-82. http://dx.doi.org/10.15359/ rep.16-2.4

Mundina Gómez, J. J., Ortega Andrés, F. y González Moreno, L-M. (1999). Juegos de Lucha, una metodología práctica para superar las acciones de contacto directo en rugby. Actas al IV Congrès de Ciències de l'Esport, l'Educació Física i la Recreació. I.N.E.F.C.-Lleida. Universitat de Lleida.

Ochoa, E. y De la Puente, Mª L. (1991). Trastornos por ansiedad. En F. Fuentenebro y C. Vázquez, Psicología Médica, Psicopatología y Psiquiatría. Vol. II: Psiquiatría y Psicología Clínicas. (pp. 709-727). Madrid: Interamericana-McGraw-Hill.

Ortiz Camacho, M. M., Delgado Noguera, M. A. y Linares Girela, D. (2000). Descripción y análisis de algunas destrezas comunicativas no verbales. Estudio de casos en la formación inicial del maestro especialista en EF. En J. L. Hernández Vázquez, Mejora del proceso de EnseñanzaAprendizaje en E.F. y Deporte. Serie ICd de investigación en Ciencias del Deporte, $n^{\circ} 28$. (pp. 53-87) Madrid: Ministerio de Educación Cultura y Deporte.

Oxford Dictionaries (n. d.). Oxford English Dictionary. http://oxforddictionaries.com/

Parks, S. E., Housemann, R. A. y Brownson, R. C. (2003). Differential correlates of physical activity in urban and rural adults of various socioeconomic backgrounds in the United States. Journal of Epidemiol Community Health, (57), 29-35. http://jech.bmj.com/content/57/1/29.full.pdf

Parlebas, P. (2001). Juegos, deportes y sociedades: léxico de praxiología motriz. Barcelona: Paidotribo.

Pease, A. y Pease, B. (2006). El lenguaje del cuerpo. Barcelona: Amat.

Pérez Turpin, J. A. y Suárez Llorca, C. (2006). Fundamentos y generalidades del baloncesto. Alicante: Editorial Club Universitario.

Petersen, W., Braun, C., Bock, W., Schmidt, K., Weimann, A., Drescher, W., Eiling, E., Stange, R., Fuchs, T., Hedderich, J. y Zantop, T. (2005). A controlled prospective case control study of a prevention training program in female team handball players: the German experience. Archives of Orthopaedic \& Trauma Surgery, 125(9), 614-621.

http://www.springerlink.com.accedys.udc.es/content/ vtbpqmvq3eewtcqx/

Peys, J-P. (1991). Rugby total et entraînement. Paris: Vigot.

Pires de Sousa, N. C. y Caramaschi, S. (2011). Contato corporal entre adolescentes através da dança de salão na escola. Motriz, Journal of Physical Education, 17(4), 618-629. http://www.scielo.br/ $\mathrm{pdf} / \mathrm{motriz} / \mathrm{v} 17 \mathrm{n} 4 / \mathrm{a} 06 \mathrm{v} 17 \mathrm{n} 4 . \mathrm{pdf}$ 
Porto Editora (n.d.). Dicionário da Língua Portuguesa da Porto Editora - com Acordo Ortográfico. http://www.infopedia.pt/lingua-portuguesa/;jsessionid=nrglSNeyrqsBrWhFbMa7sA

Preboth, M. (2000). Physical activity and fitness in schools. American Family Physician, (62), 23642366.

Real Academia Española. (2001). Diccionario de la Lengua Española $\left(22^{a}\right.$ ed). Madrid. http://www. rae.es/rae.html

Real Academia Galega (2012). Diccionario da Real Academia Galega. www.realacademiagalega. org/dicionario\#inicio.do

Roffé, M. (1999). Los miedos de los futbolistas. Lecturas: E.F. y Deportes, 4(16). http://www. efdeportes.com/efd16/miedos.htm

Roquette, J. (1994). Sistematizaçâo e análise das técnicas de controlo das quedas no judo (Ukemis). Ludens, 14(2), 45-53.

Rugby Football Union (1985). Even Better Rugby. Middlesex: Rugby Football Union.

Ruiz Munuera, F. C. (2003). Planteamiento didáctico. Iniciación al Rugby-imagen para $1^{\circ}$ de Bachillerato. En A. Ruiz, I. Perelló, N. Caus, y F. Ruiz (Eds.), Temario para la preparación de oposiciones. Educación Física: Aplicaciones Didácticas. Sevilla: MAD-Eduforma.

Rumin, J-P. (2006). La escuela de Rugby: 33 juegos para niños de 6 a 11 años. Buenos Aires: Stadium.

Rutherford, D. (1993). The complete book of Minirugby. London: Partridge Press.

Salgado López, J. I. y Rovira Bahillo, G. (2004). Análisis de los problemas psico-afectivos derivados del contacto en deportes sociomotrices de colaboración-oposición. Actas al III de Congreso de la Asociación Española de Ciencias del Deporte. Facultad de Ciencias de la Actividad Física y el Deporte. Universitat de València. València.

Salgado López, J. I., Eslava Oriol, I., Montes Lasheras, J. M. y Mariño Pego, C. (2003). Factores a tener en cuenta en la enseñanza de tareas motrices que impliquen contacto físico. Revista de Educación Física, (91), 27-38.

Salgado López, J. I. (2014). As emoções como condicionante didático no ensino dos deportes sociomotores de colaboração-oposição: estudo dos problemas afetivos ligados ao contacto en rugby, andebol e voleibol. Tesis doctoral. Universidade da Corunha. https://ruc.udc.es/dspace/ handle/2183/13840

Santos, S. G. y Melo, S. I. L. (2003). Os "ukemis" e o judoca: significado, importância, gosto e desconforto. Revista Brasileira de Cineantropometria \& Desempenho Humano, 5(2), 33-43.

Severinsen, G. (2011). But sport is good for you: Exploring the complexities of keeping children in sport and shaping a curriculum of possibilities. En G. Dodd (Ed.), Edited Proceedings of the 27th ACHPER International Conference (pp. 255-265). Adelaide: ACHPER National.

Short, S. E., Reuter, J., Brandt, J., Short, M. W. y Kontos, A. P. (2004). The relationships among three components of perceived risk of injury, previous injuries and gender in contact sport athletes. Athletic Insight: The Online Journal of Sport Psychology, 6(3).

Silva, J. M. (2006). Psychological aspects in the training and performance of team handball athletes. En J. Dosil (Eds.), The Sport Psychologist's Handbook: A Guide for Sport-Specific Performance Enhancement (pp. 211-243) West Sussex: John Wiley \& Sons Ltd.

Siquier, S. (1987). El factor miedo en el aprendizaje de tareas motrices (una reflexión al enseñar tareas motrices al niño). Revista de Educación Física, (15), 26-30.

Slobounov. S. M. (2008). Injuries in athletics: causes and consequences. New York: Springer.

Snyder, E. E. (1990). Emotion and sport: A case study of collegiate women gymnasts. Sociology of sport Journal, (7), 254-270.

Sommer, R. (1974). Espacio y comportamiento individual. Madrid: Instituto de Estudios de Administración Local.

SPARC (2006). Stay and play. http://www.sportnz.org.nz/en-nz/young-people/Guidelines-Resources/ 
(2007). It all about children and young people. Implementing a child/young person centered philosophy in sport and recreation.www.sportnz.org.nz/ Documents/Young\%20People/Its_all_ about_children_V2.pdf

Taylor, J. y Kress, J. (2006). Psychology of cycling. En J. Dosil (Eds.), The Sport Psychologist's Handbook. A Guide for Sport-Specific Performance Enhancement (pp. 325-350). West Sussex: J. Wiley \& Sons Ltd.

Teixeira Vaz, L. M. (2001). ¿Que formação para o novo jogador de rugby?. Lecturas: educación física y deportes, 7(42). http://www.efdeportes.com/efd42/rugby.htm

Thomas, J. R., Launder A. G., y Nelson J. K. (2001). Play Practice: The Games Approach to Teaching and Coaching Sports. Champaign. Illinois: Human Kinetics.

Torres Tobío, G. (1998). El conocimiento didáctico del contenido en la enseñanza de una técnica deportiva en Balonmano: El lanzamiento en salto con caída desde el extremo. La perspectiva de los expertos, entrenadores y jugadores. Tesis doctoral inédita. Universidade da Coruña.

Treanor, L., Graber, K., Housner, L. y Wiegand, R. (1998). Middle school student's perceptions of coeducational and same-sex physical education classes. Journal of Teaching Physical Education, (18), 43-56.

Troya Montañez, Y. y Cuéllar Moreno, Ma. J. (2008). Tratamiento de la danza en el diseño curricular de educación secundaria para la Comunidad Autónoma de Canarias. Actas al IV Congreso Internacional y XXV Nacional de Educación Física. Córdoba. http://www.uco.es/ IVCongresoInternacionalEducacionFisica/congreso/Documentos/001-044-108-003-001.html

Turrán Eguren, N. (2011). Problemas psico-afectivos derivados del contacto corporal. Revista Arista Digital, (15), 8-38. http://www.afapna.com/aristadigital/archivos_revista/2011_diciembre_0.pdf

Usero Martín, F. (1993). Rugby. Entrenamiento y juego. Madrid: Campomanes.

Viviani Brochado, M. M. (2002). O medo no esporte. Motriz, 8(2), 69-77. http://www.rc.unesp.br/ib/ efisica/motriz/08n2/Brochado.pdf

Walker, N., Thatcher, J. y Lavallee, D. (2010 ). A preliminary development of the Re-Injury Anxiety Inventory (RIAI). Physical Therapy in Sport, (11), 23-29.

Wehmeier, S. (2000). Oxford Advanced Learner's Dictionary of current English. Oxford University Press.

Wuo Pereira, D. (2010). Um olhar sobre a complexidade da escalada na educação física, na perspectiva de Edgar Morin. Dissertação de Mestrado. Universidade São Judas Tadeu. Brasil. http://www.usjt.br/biblioteca/mono_disser/mono_diss/2011/128_pereira.php

Yagüe Cabezón, J. M. y Lorenzo Caminero, F. (1997). Fútbol. Una propuesta curricular a través del juego. Unidades didácticas para Secundaria VII. Unidades Didácticas de Aplicación. Barcelona: INDE.

Young, D. R., Johnson, C. C., Steckler, A., Gittelsohn, J., Saunders, R. P., Saksvig, B.I., Ribisl, K. M., Lytle, L.A. y McKenzie, T. L. (2006). Using formative research to develop intervention programs to increase physical activity in adolescent girls. Health education \& behavior, 33(1), $97-111$.

Zubiaur González, M. y Gutiérrez Santiago, A. (2003). El miedo en el aprendizaje motor. Apunts: Educació Física i Esports, (72), 21-26. https://www.raco.cat/index.php/ApuntsEFD/article/ view/301342/0 